\section{Microalbuminuria y excreción urinaria de albúmina en la práctica clínica}

\author{
RODRIGO TAGLE, FERNANDO GONZÁLEZ², MÓNICA ACEVEDO ${ }^{3}$
}

\section{Microalbuminuria and urinary albumin excretion in clinical practice}

Microalbuminuria is a new tool in the management of patients with diabetes mellitus or hypertension. Microalbuminuria is an easily measured biomarker in a urine sample. Urinary albumin to creatinine ratio in first morning urine sample correlates with 24 hours urinary albumin excretion, but it is easier to obtain, and can identify hypertensive or diabetic patients with high risk for cardiovascular events. Therapeutic interventions such as renin angiotensin system blockade have demonstrated their usefulness in reducing urinary albumin excretion in clinical studies. It would be advisable to incorporate urinary albumin to creatinine ratio to the routine clinical monitoring of patients with cardiovascular risk, such as those with hypertension and diabetes mellitus.

(Rev Med Chile 2012; 140: 797-805).

Key words: Albuminuria; Creatinine; Diabetes mellitus; Hypertension.

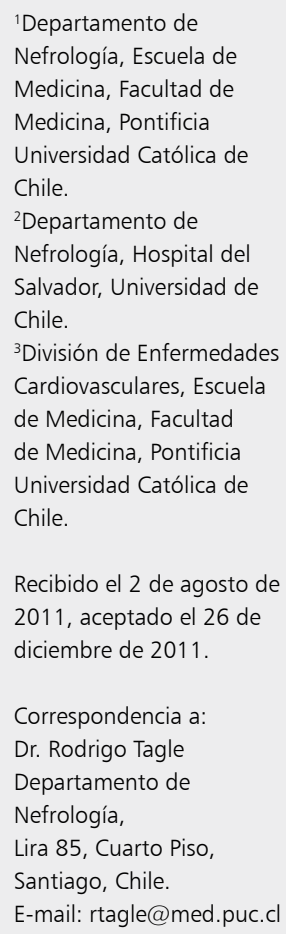

E l aumento de la prevalencia de las enfermedades cardiovasculares (ECV) y renales crónicas (ERC) trae, como consecuencia, la necesidad de buscar marcadores tempranos que sean capaces de identificar sujetos de alto riesgo de sufrir complicaciones cardiovasculares (CV) y/o renales.

Uno de estos marcadores es la excreción urinaria de albúmina (EUA) en cantidades anormales, también llamada microalbuminuria (MA). La MA ha ido ganando terreno en el ámbito del reconocimiento anticipado de individuos en riesgo como pacientes con diabetes mellitus tipo 2 (DM2) o con hipertensión (HTA).

Los objetivos de este artículo son revisar: a) en qué consiste y cómo se produce la MA; b) cómo se debe medir; c) las bases científicas de su uso clínico y d) los estudios clínicos aleatorios de prevención o reducción de la MA en pacientes con DM2 o con HTA.

\section{Definición de MA}

La EUA en una población sana corresponde entre 1,5 a $20 \mu \mathrm{g} / \mathrm{min}$, estando el percentil 90 en alrededor de $30 \mathrm{mg} / 24 \mathrm{hrs}$. El "dipstick" rutinario del examen de orina puede detectar concentraciones de albúmina en niveles superiores a $30 \mathrm{mg} / \mathrm{dL}$, que corresponde a una EUA de $300 \mathrm{mg} / 24 \mathrm{hrs}$. Todo lo que se sitúe por sobre este nivel de excreción se denomina proteinuria. En cambio, MA es definida como el rango intermedio entre lo normal y proteinuria, así MA corresponde a una EUA entre 30 a $300 \mathrm{mg} / 24 \mathrm{hrs}^{2,3}$ (Tabla 1 ).

\section{Patogenia de la MA}

La barrera de filtración glomerular, compuesta por células endoteliales, membrana basal y células podocitarias, impide que cantidades mayores a $2.000 \mathrm{mg}$ de albúmina, pasen diariamente al espacio de Bowman. La mayor parte de ella (99\%) es reabsorbida y degradada en los túbulos proximales, excretándose menos de $30 \mathrm{mg} / \mathrm{día}^{4}$ (Figura 1).

Una pequeña cantidad de fragmentos de albúmina puede aparecer en la orina provenientes de la degradación tubular, pero éstos no son detectados con los métodos utilizados en clínica ${ }^{5}$. 
Se han planteado mecanismos relacionados a trastornos hemodinámicos y celulares, no excluyentes entre sí, que tratan de explicar el por qué en algunas patologías aumenta la $\mathrm{EUA}^{6}$.

Tabla 1. Definición de microalbuminuria

\begin{tabular}{|c|c|c|c|c|}
\hline & \multicolumn{4}{|c|}{$\begin{array}{c}\text { Tipos de recolección urinaria y } \\
\text { unidades de medición }\end{array}$} \\
\hline & & tinal & 24 horas & Nocturna \\
\hline & $\underset{\mathbf{C r}}{\mathbf{m g} / \mathrm{g}}$ & $\underset{\mathrm{Cr}}{\mathrm{mg} / \mathrm{mmol}}$ & mg/24 hrs & $\mathbf{m g} / \mathbf{m i n}$ \\
\hline Normal & $\leq 29$ & $<3$ & $<30$ & $<20$ \\
\hline Microalbuminuria & $30-299$ & $3-29$ & $30-299$ & $20-199$ \\
\hline Proteinuria & $\geq 300$ & $\geq 30$ & $\geq 300$ & $\geq 200$ \\
\hline
\end{tabular}

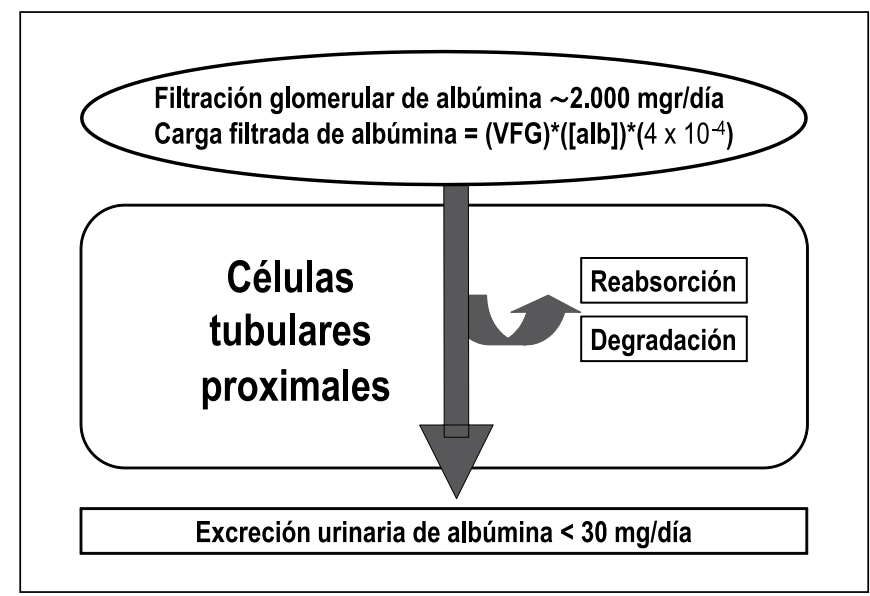

Figura 1. Excreción urinaria de albúmina.

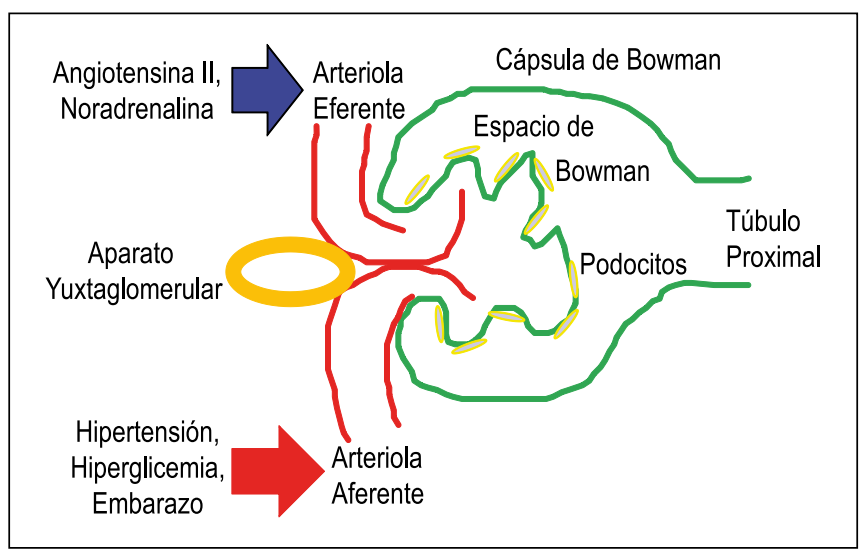

Figura 2. Factores de hipertensión intraglomerular.

\section{Trastornos hemodinámicos}

En condiciones normales la presión intraglomerular depende del tono de las arteriolas aferente (AA) y eferente (AE). En entidades con desarrollo de hipertensión intraglomerular, se produciría mayor filtración de albúmina y luego mayor EUA ${ }^{7}$.

En los diabéticos, en que se encuentra activado el sistema renina angiotensina (SRA), se produce una vasoconstricción preferentemente de la AE, y como consecuencia, hipertensión intraglomerular ${ }^{8}$. Fenómeno que se exacerba con la vasodilatación de la AA que ocurre en descompensaciones metabólicas.

En hipertensos, en la medida que se desarrolla arteríoloesclerosis en las $\mathrm{AA}$, se interfiere con la regulación de la presión intraglomerular $y$, por ende, mayor EUA'.

Así entonces, cualquier enfermedad en que se altere el equilibrio de los tonos de las $\mathrm{AA}$ y $\mathrm{AE}$, predispondrá a la aparición de albuminuria ${ }^{10,11}$ (Figura 2).

\section{Trastornos celulares}

Diversos estudios han demostrado que sustancias derivadas del endotelio son capaces de dañar la célula podocitaria, célula filtro clave, que normalmente impide el paso de albúmina ${ }^{12,13}$. Ese daño originaría un ensanchamiento de los poros del podocito con la subsiguiente pérdida de albúmina. En este contexto, se ha demostrado que quienes tienen una disfunción endotelial presentan $\mathrm{MA}^{14,15}$.

Hasta ahora no se conoce claramente el correlato morfológico del estado de MA en DM2 o HTA.

\section{Medición de MA}

Inicialmente, la EUA se medía en orina de $24 \mathrm{~h}$ o en una muestra matinal expresada como $\mathrm{mg} / \mathrm{ml}$. Estas mediciones presentan múltiples inconvenientes desde la recolección de toda la orina de un día hasta el efecto de la dilución o concentración urinaria. Por ello, se 
ha propuesto que la determinación se realice en una muestra de orina matinal, efectuando en ella la medición de albúmina y creatinina, y que los resultados se expresen como el cuociente albuminuria/creatininuria, en $\mathrm{mg} / \mathrm{g}^{16}$. Este cuociente tiene una excelente correlación con la medición tradicional en orina de $24 \mathrm{~h}^{2,3,17}$. En caso de haberse expresado en $\mathrm{mg} / \mathrm{mmol}$, puede derivarse a $\mathrm{mg} / \mathrm{g}$ al multiplicarse por $8,84^{18}$.

Por otro lado, en la actualidad las cintas reactivas, diseñadas especialmente para su medición, permiten medir la EUA con mucha confiabilidad y sensibilidad, comparado con técnicas más sofisticadas como la nefelometría ${ }^{19}$.

Las condiciones que pueden incrementar la EUA incluyen: infección del tracto urinario, insuficiencia cardiaca congestiva (ICC), embarazo, ejercicio extenuante, fiebre, posición de pie prolongada, consumo excesivo de alcohol, sobrecarga salina o proteica, deficiente control de la glicemia y contaminación con flujo cervical ${ }^{20}$.

La albúmina en la orina es estable a temperaturas normales y los procesos de almacenamiento o tiempo de éste (menos de 7 días) no incrementan los errores de las técnicas actuales ${ }^{1,21}$. No obstante lo anterior, la MA presenta una variabilidad intraindividual de un día a otro de hasta casi $40 \%$, por lo que el diagnóstico de MA debe confirmarse en una segunda determinación, de preferencia en ausencia de las condiciones que incrementan la EUA.

\section{MA como marcador de riesgo $\mathrm{CV}$ y renal}

\section{Diabetes Mellitus tipo 2}

En 1984, Mogensen y col, describieron un significativo aumento en la mortalidad cardiovascular en pacientes con DM2 que presentaban MA. En ese estudio, la mayor tasa de muertes de causa CV fue en aquellos diabéticos con EUA más elevada, aun antes de desarrollar ERC ${ }^{22}$. Posteriormente, varios estudios transversales, retrospectivos y prospectivos en pacientes con DM2 han corroborado un incremento tanto en la incidencia de ECV como de mortalidad CV en aquellos pacientes con MA, independiente de la presencia de otros factores de riesgo cardiovasculares (FRCV), y que $60 \%$ de las muertes en DM2 con MA ocurre por causas $\mathrm{CV}$, tanto cardiacas como cerebrovasculares (AVE) ${ }^{23-25}$.

El estudio HOPE (Heart Outcomes Prevention Evaluation) corroboró las diferencias de riesgo entre diabéticos con o sin MA: quienes tenían
MA presentaron un riesgo relativo $\mathrm{CV}$ mayor comparado con normoalbuminúricos y posee un valor predictivo similar a los FRCV tradicionales (Figura 3). Más aún, para un objetivo secundario como ICC, el riesgo relativo se incrementó casi cuatro veces ${ }^{11}$.

En esta misma línea, Dinneen y col, previamente habían observado hallazgos similares en un meta-análisis de 11 estudios longitudinales que incluían 2.138 pacientes con MA, con un seguimiento promedio de 6,4 años. El riesgo relativo para morbi-mortalidad cardiovascular fue 2,0 (IC 95\% $1,4-2,7$ ), y para muerte fue 2,4 (IC $95 \% 1,8-3,1)^{26}$.

$\mathrm{Al}$ analizar la MA desde el punto de vista renal, los aspectos a considerar son: proteinuria, deterioro de la velocidad de filtración glomerular (VFG) e insuficiencia renal crónica terminal (IRCt). En la mayoría de los pacientes con DM2, la nefropatía comienza con MA, la cual aumenta progresivamente hasta proteinuria y luego comienza a decaer en paralelo a la $\mathrm{VFG}^{27}$. En todo este tiempo, la probabilidad de sufrir un evento $\mathrm{CV}$ es mayor en estos enfermos que en diabéticos sin MA o proteinuria ${ }^{28}$.

Sin embargo, existe una proporción de diabéticos tipo 2 que llegan a la IRCt sin desarrollar previamente $\mathrm{MA}^{29-31}$. Así en una cohorte de 502 diabéticos con creatinina plasmática $>1,6 \mathrm{mg} / \mathrm{dL}$ o en terapia de reemplazo renal (TRR) y registros previos de EUA, sólo 2 de ellos no habían presentado $\mathrm{MA}^{32}$. En cambio, Ito y col encontraron en 1.197 diabéticos 19\% normoalbuminúricos con VFG $<60 \mathrm{ml} / \mathrm{min}^{32}$, y Tsalamandris y col, en 22 diabéticos seguidos por 8 a 14 años, observó que 6 de ellos (27\%) presentaron deterioro de la VFG sin desarrollar $\mathrm{MA}^{33}$. En consecuencia, no todos los pacientes con DM2 e IRCt han presentado previamente MA, pero sí una proporción significativa.

En estudios de pacientes con DM2 y MA la tasa de deterioro de la VFG varía entre 0 y $3,5 \mathrm{ml} / \mathrm{min} /$ año, y esta tasa tiende a cambiar según la evolución de la EUA, ya sea a normoalbuminuria, MA persistente o proteinuria ${ }^{34,35}$. En 151 pacientes con DM2 y MA del estudio Steno- 2 seguidos por 7,8 años, la tasa de deterioro de la VFG fue diferente según el cambio en la magnitud de la EUA, ya sea reversión a normoalbuminuria $(31 \%)$ de $2,3 \mathrm{ml} / \mathrm{min} /$ año, MA persistente $(38 \%)$ de $3,7 \mathrm{ml} / \mathrm{min} /$ año o proteinuria $(31 \%)$ de $5,4 \mathrm{ml} / \mathrm{min} /$ año $(\mathrm{P}<0,001)^{34}$. En 214 pacientes japoneses con DM2 y MA seguidos por 2 años, la tasa de deterioro de la VFG fue tam- 


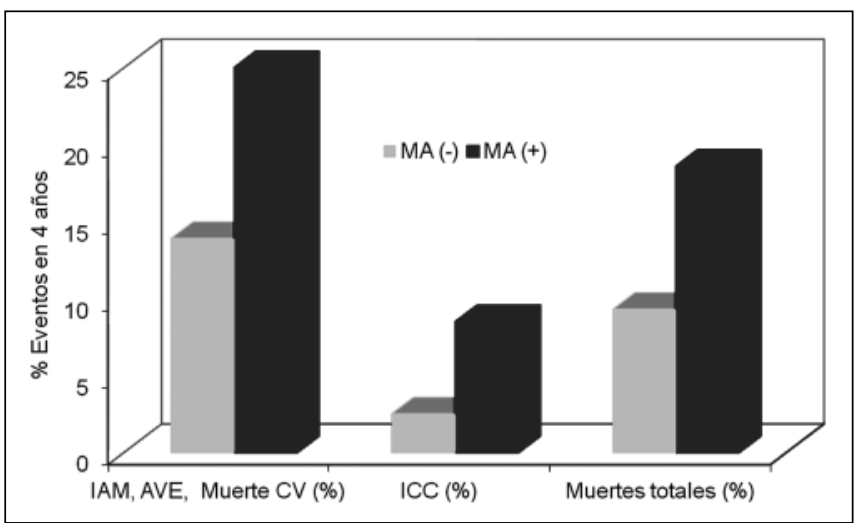

Figura 3. Eventos en el estudio Hope según MA.

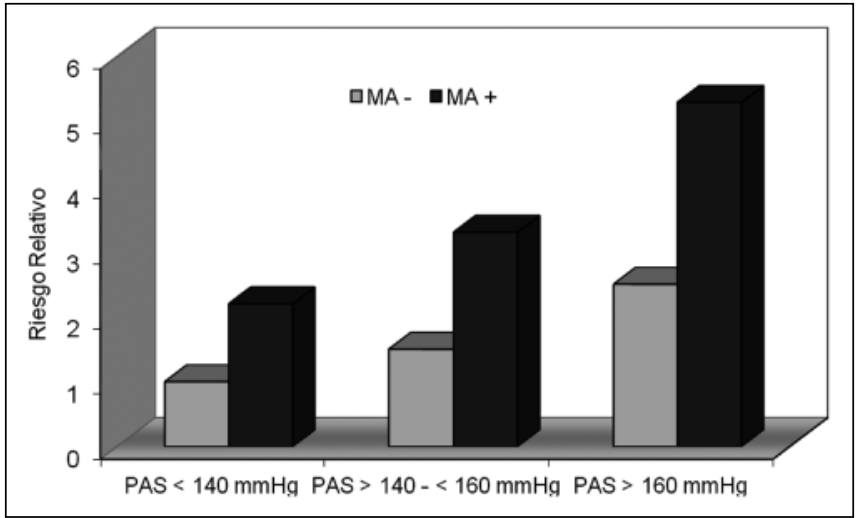

Figura 4. Riesgo relativo de Cardiopatia Coronaria según nivel de EUA.

bién diferente según la reversión a normoalbuminuria o MA persistente, $1,8 \mathrm{vs} 3,1 \mathrm{ml} / \mathrm{min} /$ año $(\mathrm{P}<0,038)^{36}$. Sin embargo, en estudios a 4 años en los indios Pima sólo se observa un cambio en la tasa de deterioro de la VFG cuando progresan de MA a proteinuria (1,0 vs $10,1 \mathrm{ml} / \mathrm{min} /$ año), pero no hay diferencia entre normoalbuminuria y $\mathrm{MA}^{37}$. Así entonces, la tasa de deterioro de la VFG tiende a ser mayor a mayor EUA, y la disminución de la EUA desde un estado de MA tiende a traducirse en un menor deterioro de la VFG.

El riesgo de desarrollar proteinuria e IRCt en DM2 con MA está claramente establecido. Así en la revisión sistemática de Newman y col, la presencia de MA le confiere un riesgo relativo de 7,5 (IC 95\% 5,2-10,9) y 3,6 (IC 95\% 1,6-8,4) respectivamente, comparados con normoalbuminúricos ${ }^{38}$. Este riesgo es 5 veces mayor si la VFG se encuentra entre 30 a $59 \mathrm{ml} / \mathrm{min}^{39}$. Eso sí en DM2, la MA como predictor de IRCt es claramente inferior a la proteinuria ${ }^{30}$.

Así, la MA en DM2 es un predictor de: deterioro de la VFG, proteinuria e IRCt; y puede considerarse un marcador de riesgo renal ${ }^{32,40}$.
Existen diversos análisis de costoefectividad de MA en DM2, que confirman su utilidad predictiva, tanto para eventos CV como para eventos renales $^{38,41-45}$.

\section{Hipertensión arterial}

En HTA se observa algo similar a lo descrito en DM2, aunque con una relación menos pronunciada. Los estudios europeos Hoorn ${ }^{46}$ y danés ${ }^{47}$ mostraron que hipertensos con MA seguidos por 5 y 10 años, elevan su riesgo CV 2,8 y 3,5 veces, respectivamente. El estudio danés demostró, por primera vez, que el hecho de ser hipertenso y tener MA, definida como EUA $>5,7 \mathrm{mg} / \mathrm{g}$, confiere un riesgo mayor de presentar un evento coronario, comparado con aquellos que sólo son hipertensos, con cualquier valor de $\mathrm{PAS}^{47}$ (Figura 4).

En el estudio LIFE (Losartan Intervention For Endpoint Reduction), efectuado en hipertensos con hipertrofia ventricular izquierda (HVI) al ECG, esta relación de MA y mayor riesgo cardiovascular fue nuevamente confirmada: la presencia de MA eleva la mortalidad y morbilidad CV en 1,7 y 1,8 veces, respectivamente ${ }^{48,49}$. Este hallazgo fue independiente del grado de HVI, edad, sexo, DM y tabaquismo ${ }^{50}$. Además al igual que en el estudio HOPE, la presencia de MA aumenta significativamente el riesgo de desarrollar ICC.

Existen escasos estudios sobre MA como marcador de riesgo renal en $\mathrm{HTA}^{51}$. Entre éstos, Bigazzi y col, en un estudio retrospectivo de 141 hipertensos seguidos por hasta 7 años reportó que el deterioro de la VFG $(12,1 \mathrm{vs} 7,1 \mathrm{ml} /$ min, $\mathrm{P}<0,03)$ y los eventos CV $(21,3$ vs $2,3 \%, \mathrm{P}<0.0002)$ fueron mayores en aquellos con MA comparados con normoalbuminúricos ${ }^{52}$. En el estudio MAGIC (Microalbuminuria: A Genoa Investigation on Complications) con 917 hipertensos seguidos por 11,8 años, la MA se relacionó significativamente con deterioro de la VFG (RR 12,7; IC $3,6-44,9 ; \mathrm{P}<0,0001)$ y con eventos CV 
(RR 2,11; IC 1,08 -4,13; P < 0,028) $)^{53}$. Finalmente, en el estudio ONTARGET (Ongoing Telmisartan Alone and in Combination with Ramipril Global Endpoint Trial) con 27.620 pacientes mayores de 55 años con ECV clínica, 70\% con HTA, seguidos por 4,8 años, la EUA en rangos de MA mostró ser un predictor independiente de IRCt ${ }^{54}$.

La explicación de la asociación entre MA y eventos CV en HTA, pareciera deberse a que la MA se asocia a los FRCV tradicionales y no tradicionales, ya que comparte mecanismos patogénicos, tales como: disfunción endotelial, resistencia a la insulina, inflamación sistémica de bajo grado, disfunción renal y elevación de la $\mathrm{PA}^{6}$. A pesar de la estrecha relación entre MA y los FRCV, la asociación entre MA y ECV se mantiene en los análisis multivariados ajustados por los FRCV tradicionales. Asi por ejemplo, el estudio PREVEND (Prevention of Renal and Vascular End-stage Disease) que incluyó más de 40.000 sujetos, las causas de MA, definida como $20-200 \mathrm{mg} / \mathrm{L}$, fueron principalmente DM2 e HTA ${ }^{55}$. En este estudio: la mortalidad CV y el deterioro de la VFG se correlacionaron con la magnitud de la EUA, y la EUA se correlacionó con el índice de masa corporal, la excreción urinaria de sodio y la edad ${ }^{56}$.

En hipertensos, la MA se asocia a daño de órganos blanco, en particular HVI y aterosclerosis carotidea (AC); y a mayor carga de $\mathrm{PA}^{57}$. En hipertensos italianos se observó que 25\% de aquellos con HVI tenían MA, pero si a la HVI se le suma $\mathrm{AC}$, el porcentaje con MA se eleva a un $46 \%{ }^{58}$. Por otro lado, la asociación de MA e HTA implica habitualmente ciertas características como ser "non-dipper" (ausencia de descenso nocturno) y "sal sensible", condiciones, ambas, relacionadas a mayor riesgo $\mathrm{CV}^{59}$. En hipertensos tratados, la aparición de MA se relaciona al nivel inicial de EUA, y a los niveles durante el seguimiento de la PAS y glicemia de ayunas ${ }^{60}$.

Así, la MA en HTA pudiese legítimamente considerarse como un marcador integral del riesgo $\mathrm{CV}^{61}$, y debido a su relación a daño de órgano blanco, se le recomienda utilizar como marcador de éste, en la estratificación de un hipertenso ${ }^{62}$.

\section{Intervenciones terapéuticas que reducen la MA}

Diabetes Mellitus tipo 2

En el estudio BENEDICT (Bergamo Nephrologic Diabetes Complications Trial), con 1.200 diabéticos tipo 2 e hipertensos sin MA seguidos por 3,6 años, se comparó el efecto sobre la aparición de MA de 4 esquemas de antihpertensivos: un inhibidor de enzima convertidora de angiotensina (IECA), un bloqueador de canales de calcio no-dihidropiridínico (verapamilo), ambos combinados o ninguno de los dos más placebo, todos asociados, según necesidad, a otros fármacos antihipertensivos para lograr una PA de 120/80 $\mathrm{mmHg}^{63}$. Los resultados mostraron que aparecía MA en: 5,7\% del grupo IECA, 6,0\% del grupo combinado, $11,9 \%$ del grupo verapamilo y $10,0 \%$ del grupo placebo. Se desprende que no basta con controlar la HTA para evitar la aparición de MA, sino que parece primordial el bloquear el SRA.

En concordancia con este estudio, están los estudios ADVANCE y ROADMAP. En el estudio ADVANCE (Action in Diabetes and Vascular Disease: Preterax and Diamicron MR Controlled Evaluation) se logró una reducción del riesgo relativo de desarrollar MA de 21\% (IC 95\% 14-27, $\mathrm{P}<0,0001$ ) en un período de 4,3 años, utilizando un IECA como perindopril comparado con una estrategia antihipertensiva sin bloqueo del SRA ${ }^{64}$. El estudio ROADMAP (Randomized Olmesartan And Diabetes Microalbuminuria Prevention Study), por su parte, demostró que diabéticos tipo 2 que reciben olmesartan, respecto de quienes reciben otra intervención antihipertensiva que no incluye el bloqueo del SRA, retardan significativamente la aparición de MA aún cuando todos logren un excelente control de la PA (Tabla 2$)^{65}$.

En diabéticos, la reducción o normalización de la MA debiese ser un objetivo terapéutico. En el estudio IRMA 2 (IRbesartan MicroAlbuminuria Type 2 Diabetes Mellitus), con diabéticos hipertensos con MA seguidos por 2 años, se encontró que irbesartan, fue más eficaz que otras terapias antihipertensivas sin bloqueo del SRA en evitar la aparición de proteinuria, 5,2\% vs $15 \%{ }^{35}$. Más aún, el tratamiento con irbesartan 300 mg logró

Tabla 2. Estudios Randomizados de prevención de la MA en diabéticos tipo 2

\begin{tabular}{|lcc|}
\hline Estudio & Tratamiento & Placebo \\
BENEDICT (3,6 años) & $5,7 \%$ & $10 \%$ \\
ADVANCE (4,6 años) & $19,6 \%$ & $23,6 \%$ \\
ROADMAP (3,2 años) & $8,2 \%$ & $9,8 \%$ \\
\hline
\end{tabular}


que un tercio de los sujetos normalizaran su EUA.

En el estudio ADVANCE, en 1.441 diabéticos con MA, el bloqueo del SRA con un IECA redujo la progresión a proteinuria $(6,2 \%$ vs $9,0 \%$ $\mathrm{p}=0,0074$, y aumentó la regresión a normoalbuminuria $(55,3 \% \text { vs } 49,1 \% \mathrm{p}=0,0067)^{39}$.

Debe resaltarse que cuando un diabético pasa de un estado de MA a proteinuria, aumenta significativamente el riesgo de muerte y eventos $\mathrm{CV}$, lo que se observó en los estudios UKPDS y ADVANCE $^{27,39}$.

\section{Hipertensión arterial}

El estudio LIFE permitió observar que un aumento o disminución de la EUA al año de seguimiento predice los eventos CV. Así, la disminución de la EUA intra-tratamiento, se relaciona a una reducción de los eventos $\mathrm{CV}$, tanto en hipertensos con o sin DM2 (Tabla 3) ${ }^{66}$.

\section{Estudios en Chile}

En Concepción, se encontró una prevalencia de $24 \%$ de MA definida como $>30 \mathrm{mg} / \mathrm{g}$, en un estudio transversal de 27.894 pacientes adultos (65\% mujeres), de los cuales 58,5\% se controlaban en el programa de salud CV. La MA se correlacionó con una VFG estimada menor a $60 \mathrm{ml} / \mathrm{min}^{67}$.

En Santiago, en un análisis retrospectivo de 269 consultantes en los cuales se le solicitó MA en un año calendario, resultó positiva en 20,8\%. La MA se correlacionó con el número de FRCV (Dr. E. Lorca. Comunicación personal).

En la Encuesta Nacional de Salud 2009 se midió la MA, pero estos resultados aún no se conocen.

\section{Guías clínicas chilenas y MA}

La guía de HTA recomienda su medición como marcador de daño de órgano blanco, y la guía de DM menciona que: "La determinación de MA persistente es un índice de nefropatía incipiente". Las actuales tablas de riesgo coronario en DM considera que deben sumarse 3 puntos a la estratificación de Framingham si presenta MA, confiriéndole un mayor riesgo ${ }^{68}$.

La guía de ERC aconseja su evaluación siempre en el contexto de otros factores de riesgo renal y $\mathrm{CV}$, y en caso de resultar positiva efectuar una estrategia terapéutica multifactorial que controle los FRCV ${ }^{69}$.
Tabla 3. Eventos CV según EUA basal y al año en el estudio LIFE

\begin{tabular}{|ccccc|}
\hline $\begin{array}{c}\text { EUA basal } \\
(\mathbf{m g} / \mathbf{g})\end{array}$ & $\begin{array}{c}\text { EUA al 1er } \\
\text { año (mg/g) }\end{array}$ & $\begin{array}{c}\text { Muerte } \\
(\mathbf{\%})\end{array}$ & $\begin{array}{c}\text { AVE } \\
\mathbf{( \% )}\end{array}$ & $\begin{array}{c}\text { IAM } \\
\text { (\%) }\end{array}$ \\
$<11$ & $<6$ & 1,8 & 2,5 & 2,1 \\
$<11$ & $>6$ & 2,7 & 4,3 & 3,3 \\
$>11$ & $<6$ & 3,4 & 4,5 & 3,7 \\
$>11$ & $>6$ & 6,1 & 6,5 & 4,7 \\
\hline
\end{tabular}

\section{Conclusiones}

La MA, cuociente albuminuria y creatininuria, es un biomarcador fácil de medir y de bajo costo en una muestra de orina matinal. Este examen permite identificar a hipertensos y/o diabéticos con alto riesgo de sufrir ECV. Las intervenciones terapéuticas que reducen la EUA, particularmente el bloqueo del SRA, pueden disminuir el riesgo asociado a la MA. Sería aconsejable que la EUA se incorporase al seguimiento clínico de hipertensos y/o diabéticos.

\section{Referencias}

1. Keane WF, Eknoyan G. Proteinuria, albuminuria, risk, assessment, detection, elimination (PARADE): a position paper of the National Kidney Foundation. Am J Kidney Dis 1999; 33 (5): 1004-10.

2. Montanes Bermúdez R, Gracia García S, Pérez Surribas D, Martínez Castelao A, Bover Sanjuan J. Consensus Document. Recommendations on assessing proteinuria during the diagnosis and follow-up of chronic kidney disease. Nefrología 2011; 31 (3): 331-45.

3. K/DOQI clinical practice guidelines for chronic kidney disease: evaluation, classification, and stratification. Am J Kidney Dis 2002; 39 (2 Suppl 1): S1-266.

4. Glassock RJ. Is the presence of microalbuminuria a relevant marker of kidney disease? Curr Hypertens Rep 2010; 12 (5): 364-8.

5. Redon J. Measurement of microalbuminuria-what the nephrologist should know. Nephrol Dial Transplant 2006; 21 (3): 573-6.

6. Cerasola G, Cottone S, Mule G. The progressive pathway of microalbuminuria: from early marker of renal damage to strong cardiovascular risk predictor. J Hypertens 2010; 28 (12): 2357-69.

7. Zatz R, Meyer TW, Rennke HG, Brenner BM. Predominance of hemodynamic rather than metabolic factors in 
the pathogenesis of diabetic glomerulopathy. Proc Natl Acad Sci USA 1985; 82 (17): 5963-7.

8. Anderson S, Brenner BM. Pathogenesis of diabetic glomerulopathy: hemodynamic considerations. Diabetes Metab Rev 1988; 4 (2):163-77.

9. Palatini P, Mormino P, Dorigatti F, Santonastaso M, Mos L, De Toni R, et al. Glomerular hyperfiltration predicts the development of microalbuminuria in stage 1 hypertension: the HARVEST. Kidney Int 2006; 70 (3): 578-84.

10. Bianchi S, Bigazzi R, Campese VM. Microalbuminuria in essential hypertension: significance, pathophysiology, and therapeutic implications. Am J Kidney Dis. 1999; 34 (6): 973-95.

11. Gerstein HC, Mann JF, Yi Q, Zinman B, Dinneen SF, Hoogwerf B, et al. Albuminuria and risk of cardiovascular events, death, and heart failure in diabetic and nondiabetic individuals. JAMA 2001; 286 (4): 421-6.

12. Satchell SC, Tooke JE. What is the mechanism of microalbuminuria in diabetes: a role for the glomerular endothelium? Diabetologia 2008; 51 (5): 714-25.

13. Haraldsson B, Nyström J, Deen WM. Properties of the Glomerular Barrier and Mechanisms of Proteinuria. Physiological Reviews 2008; 88 (2): 451-87.

14. Stehouwer CD. Endothelial dysfunction in diabetic nephropathy: state of the art and potential significance for non-diabetic renal disease. Nephrol Dial Transplant 2004; 19 (4): 778-81.

15. Pedrinelli R, Penno G, Dell’Omo G, Bandinelli S, Giorgi D, Di Bello V, et al. Microalbuminuria and transcapillary albumin leakage in essential hypertension. Hypertension 1999; 34 (3): 491-5.

16. Levey AS, Eckardt KU, Tsukamoto Y, Levin A, Coresh J, Rossert J, et al. Definition and classification of chronic kidney disease: a position statement from Kidney Disease: Improving Global Outcomes (KDIGO). Kidney Int 2005; 67 (6): 2089-100.

17. Eknoyan G, Hostetter T, Bakris GL, Hebert L, Levey AS, Parving $\mathrm{HH}$, et al. Proteinuria and other markers of chronic kidney disease: a position statement of the national kidney foundation (NKF) and the national institute of diabetes and digestive and kidney diseases (NIDDK). Am J Kidney Dis 2003; 42 (4): 617-22.

18. Wachtell $\mathrm{K}$, Ibsen $\mathrm{H}$, Olsen $\mathrm{MH}$, Borch-Johnsen $\mathrm{K}$, Lindholm LH, Mogensen CE, et al. Albuminuria and cardiovascular risk in hypertensive patients with left ventricular hypertrophy: the LIFE study. Ann Intern Med 2003; 139 (11): 901-6.

19. Gerber LM, Johnston K, Alderman MH. Assessment of a new dipstick test in screening for microalbuminuria in patients with hypertension. Am J Hypertens 1998; 11 (11 Pt 1): 1321-7.
20. Mogensen CE, Vestbo E, Poulsen PL, Christiansen C, Damsgaard EM, Eiskjaer H, et al. Microalbuminuria and potential confounders. A review and some observations on variability of urinary albumin excretion. Diabetes Care 1995; 18 (4): 572-81.

21. Gansevoort RT, Verhave JC, Hillege HL, Burgerhof JG, Bakker SJ, de Zeeuw D, et al. The validity of screening based on spot morning urine samples to detect subjects with microalbuminuria in the general population. Kidney Int Suppl 2005; 94: S28-35.

22. Mogensen CE. Microalbuminuria predicts clinical proteinuria and early mortality in maturity-onset diabetes. N Engl J Med 1984; 310 (6): 356-60.

23. Schmitz A, Vaeth M. Microalbuminuria: a major risk factor in non-insulin-dependent diabetes. A 10-year follow-up study of 503 patients. Diabet Med 1988; 5 (2): 126-34.

24. Gall MA, Borch-Johnsen K, Hougaard P, Nielsen FS, Parving HH. Albuminuria and poor glycemic control predict mortality in NIDDM. Diabetes 1995; 44 (11): 1303-9.

25. Miettinen H, Haffner SM, Lehto S, Ronnemaa T, Pyorala K, Laakso M. Proteinuria predicts stroke and other atherosclerotic vascular disease events in nondiabetic and non-insulin-dependent diabetic subjects. Stroke 1996; 27 (11): 2033-9.

26. Dinneen SF, Gerstein HC. The association of microalbuminuria and mortality in non-insulin-dependent diabetes mellitus. A systematic overview of the literature. Arch Intern Med 1997; 157 (13): 1413-8.

27. Adler AI, Stevens RJ, Manley SE, Bilous RW, Cull CA, Holman RR. Development and progression of nephropathy in type 2 diabetes: the United Kingdom Prospective Diabetes Study (UKPDS 64). Kidney Int 2003; 63 (1): 225-32.

28. Tight blood pressure control and risk of macrovascular and microvascular complications in type 2 diabetes: UKPDS 38. UK Prospective Diabetes Study Group. BMJ 1998; 317 (7160): 703-13.

29. Middleton RJ, Foley RN, Hegarty J, Cheung CM, McElduff P, Gibson JM, et al. The unrecognized prevalence of chronic kidney disease in diabetes. Nephrol Dial Transplant 2006; 21 (1): 88-92.

30. Glassock RJ. Debate: CON position. Should microalbuminuria ever be considered as a renal endpoint in any clinical trial? Am J Nephrol 2010; 31 (5): 462-5; discussion 6-7.

31. Kramer CK, Leitao CB, Pinto LC, Silveiro SP, Gross JL, Canani LH. Clinical and laboratory profile of patients with type 2 diabetes with low glomerular filtration rate and normoalbuminuria. Diabetes Care 2007; 30 (8): 1998-2000. 
32. Karalliedde J, Viberti G. Proteinuria in diabetes: bystander or pathway to cardiorenal disease? J Am Soc Nephrol 2010; 21 (12): 2020-7.

33. Tsalamandris C, Allen TJ, Gilbert RE, Sinha A, Panagiotopoulos S, Cooper ME, et al. Progressive decline in renal function in diabetic patients with and without albuminuria. Diabetes 1994; 43 (5): 649-55.

34. Gaede P, Tarnow L, Vedel P, Parving HH, Pedersen O. Remission to normoalbuminuria during multifactorial treatment preserves kidney function in patients with type 2 diabetes and microalbuminuria. Nephrol Dial Transplant 2004; 19 (11): 2784-8.

35. Parving HH, Lehnert H, Brochner-Mortensen J, Gomis $\mathrm{R}$, Andersen S, Arner P. The effect of irbesartan on the development of diabetic nephropathy in patients with type 2 diabetes. N Engl J Med 2001; 345 (12): 870-8.

36. Araki S-I, Haneda M, Koya D, Hidaka H, Sugimoto T, Isono $\mathrm{M}$, et al. Reduction in Microalbuminuria as an Integrated Indicator for Renal and Cardiovascular Risk Reduction in Patients With Type 2 Diabetes. Diabetes 2007; 56 (6): 1727-30.

37. Nelson RG, Bennett PH, Beck GJ, Tan M, Knowler WC, Mitch WE, et al. Development and progression of renal disease in Pima Indians with non-insulin-dependent diabetes mellitus. Diabetic Renal Disease Study Group. N Engl J Med 1996; 335 (22): 1636-42.

38. Newman DJ, Mattock MB, Dawnay AB, Kerry S, McGuire A, Yaqoob M, et al. Systematic review on urine albumin testing for early detection of diabetic complications. Health Technol Assess 2005; 9 (30): iii-vi, xiii-163.

39. de Galan BE, Perkovic V, Ninomiya T, Pillai A, Patel A, Cass A, et al. Lowering blood pressure reduces renal events in type 2 diabetes. J Am Soc Nephrol 2009; 20 (4): 883-92.

40. Jerums G, Premaratne E, Panagiotopoulos S, Clarke S, Power DA, MacIsaac RJ. New and old markers of progression of diabetic nephropathy. Diabetes Res Clin Pract 2008; 82 Suppl 1: S30-7.

41. Hoerger TJ, Wittenborn JS, Segel JE, Burrows NR, Imai K, Eggers P, et al. A Health Policy Model of CKD: 2. The Cost-Effectiveness of Microalbuminuria Screening. American journal of kidney diseases: the official journal of the National Kidney Foundation 2010; 55 (3): 463 73.

42. Palmer AJ, Valentine WJ, Chen R, Mehin N, Gabriel $\mathrm{S}$, Bregman B, et al. A health economic analysis of screening and optimal treatment of nephropathy in patients with type 2 diabetes and hypertension in the USA. Nephrology Dialysis Transplantation 2008; 23 (4): 1216-23.

43. Golan L, Birkmeyer JD, Welch HG. The Cost-Effective- ness of Treating All Patients with Type 2 Diabetes with Angiotensin-Converting Enzyme Inhibitors. Annals of Internal Medicine 1999; 131 (9): 660-7.

44. Incerti J, Zelmanovitz T, Camargo JL, Gross JL, de Azevedo MJ. Evaluation of tests for microalbuminuria screening in patients with diabetes. Nephrol Dial Transplant 2005; 20 (11): 2402-7.

45. Lepore G, Maglio ML, Nosari I, Dodesini AR, Trevisan R. Cost-effectiveness of two screening programs for microalbuminuria in type 2 diabetes. Diabetes Care 2002; 25 (11): 2103-4; author reply 4.

46. Jager A, Kostense PJ, Ruhe HG, Heine RJ, Nijpels G, Dekker JM, et al. Microalbuminuria and peripheral arterial disease are independent predictors of cardiovascular and all-cause mortality, especially among hypertensive subjects: five-year follow-up of the Hoorn Study. Arterioscler Thromb Vasc Biol 1999; 19 (3): 617-24.

47. Borch-Johnsen K, Feldt-Rasmussen B, Strandgaard S, Schroll M, Jensen JS. Urinary albumin excretion. An independent predictor of ischemic heart disease. Arterioscler Thromb Vasc Biol 1999; 19 (8): 1992-7.

48. Wachtell K, Olsen MH, Dahlof B, Devereux RB, Kjeldsen SE, Nieminen MS, et al. Microalbuminuria in hypertensive patients with electrocardiographic left ventricular hypertrophy: the LIFE study. J Hypertens 2002; 20 (3): 405-12.

49. Ibsen $\mathrm{H}$, Wachtell $\mathrm{K}$, Olsen $\mathrm{MH}$, Borch-Johnsen $\mathrm{K}$, Lindholm LH, Mogensen CE, et al. Does albuminuria predict cardiovascular outcome on treatment with losartan versus atenolol in hypertension with left ventricular hypertrophy? A LIFE substudy. J Hypertens 2004; 22 (9): 1805-11.

50. Olsen MH, Wachtell K, Bella JN, Palmieri V, Gerdts E, Smith G, et al. Albuminuria predicts cardiovascular events independently of left ventricular mass in hypertension: a LIFE substudy. J Hum Hypertens 2004; 18 (6): 453-9.

51. Lambers Heerspink HJ, de Zeeuw D. Debate: PRO position. Should microalbuminuria ever be considered as a renal endpoint in any clinical trial? Am J Nephrol 2010; 31 (5): 458-61; discussion 68.

52. Bigazzi R, Bianchi S, Baldari D, Campese VM. Microalbuminuria predicts cardiovascular events and renal insufficiency in patients with essential hypertension. J Hypertens 1998; 16 (9): 1325-33.

53. Viazzi F, Leoncini G, Conti N, Tomolillo C, Giachero $\mathrm{G}$, Vercelli $\mathrm{M}$, et al. Microalbuminuria is a predictor of chronic renal insufficiency in patients without diabetes and with hypertension: the MAGIC study. Clin J Am Soc Nephrol 2010; 5 (6): 1099-106.

54. Clase CM, Gao P, Tobe SW, McQueen MJ, Grosshennig 
A, Teo KK, et al. Estimated glomerular filtration rate and albuminuria as predictors of outcomes in patients with high cardiovascular risk: a cohort study. Ann Intern Med 2011; 154 (5): 310-8.

55. Hillege HL, Janssen WM, Bak AA, Diercks GF, Grobbee DE, Crijns HJ, et al. Microalbuminuria is common, also in a nondiabetic, nonhypertensive population, and an independent indicator of cardiovascular risk factors and cardiovascular morbidity. J Intern Med 2001; 249 (6): 519-26.

56. Hillege HL, Fidler V, Diercks GF, van Gilst WH, de Zeeuw D, van Veldhuisen DJ, et al. Urinary albumin excretion predicts cardiovascular and noncardiovascular mortality in general population. Circulation 2002; 106 (14): 1777-82.

57. Agrawal B, Berger A, Wolf K, Luft FC. Microalbuminuria screening by reagent strip predicts cardiovascular risk in hypertension. J Hypertens 1996; 14 (2): 223-8.

58. Pontremoli R, Viazzi F, Sofia A, Tomolillo C, Ruello N, Bezante GP, et al. Microalbuminuria: a marker of cardiovascular risk and organ damage in essential hypertension. Kidney Int Suppl 1997; 63: S163-5.

59. Hogan D, Lurbe E, Salabat MR, Redon J, Batlle D. Circadian changes in blood pressure and their relationships to the development of microalbuminuria in type 1 diabetic patients. Curr Diab Rep 2002; 2 (6): 539-44.

60. Redon J, Williams B. Microalbuminuria in essential hypertension: redefining the threshold. J Hypertens 2002; 20 (3): 353-5.

61. Pedrinelli R, Dell'Omo G, Di Bello V, Pontremoli R, Mariani M. Microalbuminuria, an integrated marker of cardiovascular risk in essential hypertension. J Hum Hypertens 2002; 16 (2): 79-89.

62. Mancia G, De Backer G, Dominiczak A, Cifkova R, Fagard R, Germano G, et al. Guidelines for the Manage- ment of Arterial Hypertension: The Task Force for the Management of Arterial Hypertension of the European Society of Hypertension (ESH) and of the European Society of Cardiology (ESC). J Hypertens 2007; 25 (6): 1105-87.

63. Ruggenenti P, Fassi A, Ilieva AP, Bruno S, Iliev IP, Brusegan $\mathrm{V}$, et al. Preventing microalbuminuria in type 2 diabetes. N Engl J Med 2004; 351 (19): 1941-51.

64. Patel A, MacMahon S, Chalmers J, Neal B, Woodward $\mathrm{M}$, Billot L, et al. Effects of a fixed combination of perindopril and indapamide on macrovascular and microvascular outcomes in patients with type 2 diabetes mellitus (the ADVANCE trial): a randomised controlled trial. Lancet 2007; 370 (9590): 829-40.

65. Haller H, Ito S, Izzo JL Jr, Januszewicz A, Katayama S, Menne J, et al. Olmesartan for the delay or prevention of microalbuminuria in type 2 diabetes. $\mathrm{N}$ Engl J Med 2011; 364 (10): 907-17.

66. Ibsen $\mathrm{H}$, Olsen $\mathrm{MH}$, Wachtell $\mathrm{K}$, Borch-Johnsen $\mathrm{K}$, Lindholm LH, Mogensen CE, et al. Reduction in albuminuria translates to reduction in cardiovascular events in hypertensive patients: losartan intervention for endpoint reduction in hypertension study. Hypertension 2005; 45 (2): 198-202.

67. Zuñiga C, Muller H, Flores M. Prevalencia de enfermedad renal crónica en centros urbanos de atención primaria. Rev Med Chile 2011; 139 (9).

68. Icaza G, Núñez L, Marrugat J, Mujica V, Escobar MC, Jiménez AL, et al. [Estimation of coronary heart disease risk in Chilean subjects based on adapted Framingham equations]. Rev Med Chile 2009; 137 (10): 1273-82.

69. Flores JC, Alvo M, Borja H, Morales J, Vega J, Zúñiga C, et al. [Clinical guidelines on identification, management and complications of chronic kidney disease]. Rev Med Chile 2009; 137 (1): 137-77. 